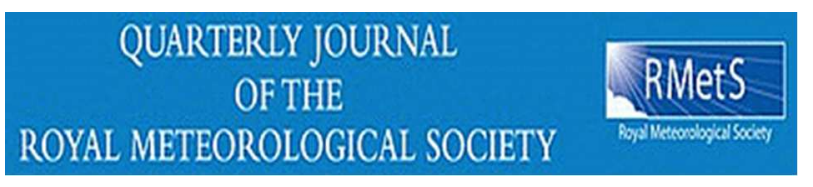

An Analytic Model of Tropical Cyclone Wind Profiles

\begin{tabular}{|r|l|}
\hline Journal: & QJRMS \\
\hline Manuscript ID: & QJ-14-0263.R3 \\
\hline Wiley - Manuscript type: & Research Article \\
\hline Date Submitted by the Author: & n/a \\
\hline Keywords: & $\begin{array}{l}\text { Tropical cyclone, wind profile, size, moist entropy distribution } \\
\text { Toumi, Ralf; Imperial College London, Physics } \\
\text { Van Kand Arnaud; Imperial College London, Physics }\end{array}$ \\
\hline &
\end{tabular}




\section{An Analytic Model of Tropical Cyclone Wind Profiles}

Tropical cyclone (TC) size is an important feature setting the extent of coastal flooding, the size of the storm surge and area threatened by landfall. The importance of TC size is demonstrated comparing Hurricanes Sandy in $2012 *$ and Bret in 1999 (Lawrence et al. 2001). As a Saffir-Simpson Hurricane Scale (Simpson and Saffir 1974) category-3 hurricane, the radius of gale-force wind of Hurricane Sandy exceeded $800 \mathrm{~km}$ prior to landfall, and the storm caused catastrophic storm surge into the

${ }^{*}$ Data available at http://www.nhc.noaa.gov/2012atlan.shtml

A physically based analytic model ( $\lambda$ model) is presented to describe the wind profile of the tropical cyclones in terms of the pressure deficit and a single shape parameter $(\lambda)$. To test the $\lambda$ model, an idealized full-physics numerical model is employed to provide wind profile samples and also to show the influence of environmental temperature and properties of initial vortices on tropical cyclone size. It is found that the $\lambda$ model provides an accurate fit of azimuthal wind profile at the top of boundary layer. In the simulations, tropical cyclone size is sensitive to the sea surface temperature, upper tropospheric temperature and initial vortex structure. The numerical model confirms the assumed Gaussian distribution with width $\lambda$ of the moist entropy in the boundary layer. A linear relationship between model cyclone size and $\sqrt{\lambda}$ is found in agreement with the $\lambda$ model. The $\lambda$ model predicts a weak relationship between tropical cyclone size and intensity as is observed. In addition, the $\lambda$ model suggests that the change in tropical cyclone size should be closely related to the angular momentum transport near the boundary layer as has been found in observations. The good agreement of the $\lambda$ model with the numerical model shows that the $\lambda$ model could be a reasonable alternative for characterizing the wind structure of tropical cyclones with only one scaling parameter. Key Words: $\quad$ tropical cyclone; wind profile; size; moist entropy distribution Received...

1. Introduction 
New Jersey and New York coastlines, and damage up to an estimated total of 50 USD billion. Hurricane Bret, on the other hand, was a more intense category-4 hurricane with a radius of gale-force wind of only $140 \mathrm{~km}$. Although the intensity of Bret is considerable, damage was reported to be relatively light, totalling an estimated 60 USD million. The difference impacts are mainly caused by the difference in TC size.

There are substantially spatial and temporal variations in TC size. For example, Brand (1972) found geographic and seasonal variations of very large and very small TCs. Merrill (1984) showed that the frequency of large TCs in the North Atlantic reaches a minimum in midsummer and a maximum in October. Furthermore, Western North Pacific TCs are significantly larger than North Atlantic TCs (Liu and Chan 1999; Chan and Chan 2012).

Despite the fact that a wide range of observed TC sizes has been recognised, the underlying factors that control both individual storm size and the climatological size variation remain mysterious. Changes in TC size are probably induced by environmental factors and the properties of initial vortices. By artificially increasing latent heating in the region of outer rainbands of simulated TCs, Wang (2009) found an outward expansion of winds, which in turn can increase the TC size. Radu et al. (2014) suggested that the increase in TC size is proportional to the surface latent heat flux by altering air-sea temperature difference. With regards to the properties of initial vortices, Xu and Wang (2010a) suggested that although the simulated TC intensity at the mature stage may be weakly determined by the initial vortex size, the simulated TC inner-core size is largely dependent on the initial vortex size. Interestingly, this idea is partly confirmed by a statistical study on TC size carried out by Dean et al. (2009), who suggested that the size of a given TC may be a function of the geometry of the precursor disturbance that serves to initiate it.

The size of a TC is usually defined with surface pressure and near-surface wind. Merrill (1984) measured size as the average radius of the outer closed isobar. In order to link the TC size with its potential destructiveness, TC size can also be defined with the near-surface wind speed (Chan and Yip 2003; Dean et al. 2009; Xu and Wang 2010a,b; Radu et al. 2014; Phibbs and Toumi 2014).
As a TC is an approximately axisymmetric system, the size can be calculated with the azimuthally averaged wind profile. Based on observational data and gradient wind equations, Holland (1980) developed one of the most commonly used tangential wind profile model (hereafter $\mathrm{H}$ model). The $\mathrm{H}$ model contains the pressure deficit from the TC centre to the ambient environment $(\Delta p)$, the Coriolis parameter and two scaling parameters, $A$ and $B . B$ is a measure describing the shape of wind profiles. An increase in $B$ indicates that the wind profile becomes more "flat" than the original. The $\mathrm{H}$ model has been successfully implemented in TC forecasting and risk models. By fitting the wind data to the $\mathrm{H}$ model one can easily calculate the TC size. However, the $\mathrm{H}$ model is partly based on a statistical fit to observational data and it is difficult to get an analytical solution to TC size by giving a threshold wind speed. A new theoretical wind profile model is presented here which overcomes these limitations.

The main goal of this paper is to derive a new TC model for the wind profile based on the Emanuel (1986, hereafter E86) airsea interaction theory. The new model should be able to provide an analytical solution for TC size, which could be beneficial to further TC size studies. To test the new theoretical model, four sets of sensitivity experiments on TC size are conducted by using a full-physics idealized numerical model. The sensitivity experiments can also show the influences of environmental temperature and properties of initial vortices on TC size, which is the other goal of this study.

The following section introduces the idealized model setup, sensitivity experiment design and the derivation of the new theoretical model. Section 3 presents the simulated TC intensity and size, and the relationship between the size changes caused by different factors and the moist entropy distribution at the top of boundary layer (TBL). Physical insights of this study are discussed in Section 4. Section 5 summarises the overall findings.

\section{Method}

\subsection{Model setup and experiment design}

In order to reduce the case-specific impacts, we utilized a fullphysics Advanced Research WRF model to simulate ideal TCs. The model was configured with two domains: a coarse mesh of 15 
$\mathrm{km}$ horizontal grid spacing and a two-way and vortex-following nested domain of $3 \mathrm{~km}$ grid spacing. The domains were square and were $4500 \mathrm{~km}(300 \times 300$ grid points $)$ and $1503 \mathrm{~km}(501 \times$ 501 points). There were 31 sigma $(\sigma)$-levels in the vertical with a higher resolution located in the boundary layer, and model top of $50 \mathrm{hPa}$. All the experiments lasted for 8 days.

All experiments were initialised with an axisymmetric vortex. The initially horizontal wind field $V_{0}(r)$ of the vortex was specified with an idealized vortex wind model following Chan and Williams (1987):

$$
V_{0}(r)=V_{m}\left(\frac{r}{R_{m}}\right) e^{\frac{1}{b}\left[1-\left(\frac{r}{R_{m}}\right)^{b}\right]}
$$

where $r$ is the radius from the vortex centre, $V_{m}$ the initial maximum wind, $R_{m}$ the radial position of $V_{m}$, and $b$ a factor that can determine the shape of the wind profile. In all of the experiments, $b$ was set as 0.33 (Hill and Lackmann 2009). Vertical structure was introduced to the horizontal wind field by decaying linearly with height (Rotunno and Emanuel 1987):

$$
V(r, z)=V_{0}(r) \frac{z^{*}-z}{z^{*}}
$$

In (2), $v=0$ at $z=z^{*}$, and we let $v=0$ for $z>z^{*}$ and set $z^{*}$ as $20 \mathrm{~km}$.

The environmental sounding profile was specified as the mean tropical sounding in "hurricane season" (July-October) of Jordan (1958), and the background flow was set as $0 \mathrm{~ms}^{-1}$. After an initial vortex was added into the environment, the temperature field was computed from thermal wind balance, geopotential heights were set in terms of gradient wind balance, and the pressure perturbations were calculated by the hydrostatic equation (Kwok and Chan 2005). Note that these adjustments may change the initial central pressure if the initial wind field is changed. At any given $\sigma$-level the relative humidity was set as a constant value with Jordan's sounding, so the temperature and pressure perturbation induced a specific humidity adjustment.

The idealized experiments were conducted over an oceanonly domain. Model experiments were performed on an $f$ plane, and the Coriolis parameter $f$ was set constant at $20^{\circ} \mathrm{N}$ $\left(f \approx 5 \times 10^{-5} \mathrm{~s}^{-1}\right)$. All experiments used the following setup:
Tiedtke cumulus parametrization scheme (Tiedtke 1989; Zhang et al. 2011) on the 15-km grids only, WSM 6-class graupel scheme (Hong and Lim 2006) of microphysical processes, the Rapid Radiative Transfer Model scheme (Mlawer et al. 1997) to estimate the effects of long-wave radiation and Dudhia (1989) implementation for short-wave radiation. The surface layer used similarity scheme based on Monin-Obukhov with CarslonBoland viscous sub-layer and standard similarity functions, along with Yonsei University scheme (Hong and Lim 2006) for parametrization of processes in the planetary boundary layer. The lateral boundary condition for the outermost domain was specified by Jordan's sounding.

In the control experiment (CTRL), the sea surface temperature (SST) was fixed at $28^{\circ} \mathrm{C}$ and the initial vortex maximum wind speed was $20 \mathrm{~m} \mathrm{~s}^{-1}$ at a radius of $75 \mathrm{~km}$ from the vortex centre. Four sets of sensitivity experiments were designed to investigate the sensitivity of TC size to environmental temperature and initial vortex parameters (Table 1). Emanuel et al. (2013) showed that, according to the reanalysis data and weather station records, in the past 30 years there was a $2 \mathrm{~K}$ decrease in tropical tropopause temperature, and this cooling influenced the Atlantic hurricane activity. On the other hand, Hill and Lackmann (2011) found that projected upper tropospheric warming in the next 100 years might change the TC intensity. However, how the TC size responds to the upper-tropospheric temperature has not been studied specifically. The near-surface heat exchange is important to TC change and we consider the importance of SST to quantify its impact on TC size. The size of a mature TC may also be determined by the initial size of the TC (Rotunno and Emanuel 1987; Cocks and Gray 2002; Xu and Wang 2010a; Chan and Chan 2014b). In each experiment, only one of the SST, upper tropospheric (150-300 $\mathrm{hPa}$ ) temperature $\left(T_{U T}\right), V_{m}$ and $R_{m}$ in the initial wind field was changed (Figure 1).

Even though we fixed the $b$ parameter in (1), the changes in $V_{m}$ or $R_{m}$ can still result in the shape change in the wind profile. The $\mathrm{H}$ model is employed here to describe these initial shape changes. With the 3 fitting parameters the $\mathrm{H}$ model gives excellent approximations (Pearson correlation coefficient, $R^{2}>0.99$ ) to the numerical model profiles. The $B$ values and the profiles in Figure (1(b)) show that the whole wind profile is changed when 
altering $V_{m}$ and $R_{m}$ in (1). More discussion about their influence on TC size is in Section 4.

To verify the simulated cyclones are fully developed, we compared the simulated intensity with the TC maximum potential intensity (MPI) of Emanuel (1995)

$$
V_{m p i}=(1-\gamma) \sqrt{C_{p}\left(T_{s}-T_{\text {out }}\right) \frac{C_{K}}{C_{D}}\left(\ln \theta_{e}^{*}-\ln \theta_{e}\right)}
$$

where $V_{m p i}$ is the potential maximum near-surface wind speed and also the approximate MPI if defining TC intensity with nearsurface winds, $\gamma$ the coefficient reflecting the typical relationship between gradient winds and actual near-surface winds $(\gamma=$ $0.2), C_{p}$ the heat capacity at constant pressure, $T_{s}$ the ocean surface temperature, $T_{\text {out }}$ the mean outflow temperature, $C_{K}$ the exchange coefficient for enthalpy, $C_{D}$ the drag coefficient, $\theta_{e}^{*}$ the saturation equivalent potential temperature evaluated under the eyewall of a developed TC at sea level, $\theta_{e}$ the boundary layer equivalent potential temperature. Note that the coefficient $\gamma$ was not considered by Emanuel (1995). Theoretically, if a cyclone gets fully developed, its intensity should be close to but not exceed the MPI.

\subsection{The $\lambda$ model for wind speed distribution}

E86 set up an air-sea interaction theory for a steady-state TC. Based on this theory, we have obtained analytical solutions for wind speed distribution and hence TC size, which we briefly describe below.

For a steady-state axisymmetric TC over an ocean with constant temperature, we shall assume that above the boundary layer, and except in the outflow at large radii, the moist entropy $\left(s_{m}\right)$ and angular momentum per unit mass $(M)$ are conserved. They are defined as,

$$
\begin{gathered}
s_{m}=\left\{\left(1-q_{t}\right) C_{p d}+q_{t} C_{l}\right\} \ln \left(\frac{T}{T_{o}}\right) \\
-\left(1-q_{t}\right) R_{d} \ln \left(\frac{p_{d}}{p_{o}}\right)+q_{v} \frac{L_{v}}{T}-q_{v} R_{v} \ln R H \\
M=r V+\frac{1}{2} f r^{2}
\end{gathered}
$$

where $V$ is the tangential velocity, $f$ the Coriolis parameter, $C_{l}$ the heat capacity of liquid water, $C_{p d}$ the heat capacity of dry (C) 0000 Royal Meteorological Society air, $T$ the air temperature, $p_{d}$ the partial pressure of dry air, $R H$ the relative humidity, $R_{d}$ the gas constant of dry air, $R_{v}$ the gas constant of water vapor, $L_{v}$ the latent heat of vaporisation, $q_{v}$ the specific humidity of water vapor, $q_{t}=q_{v}+q_{l}$ and $q_{l}$ the specific humidity of condensate water, $T_{o}$ the arbitrary reference temperature (set as $273.15 \mathrm{~K}$ ) and $p_{o}$ the arbitrary reference partial pressure of dry air (set as $1000 \mathrm{hPa}$ ). The reader is referred to (Pauluis et al. 2010) for a derivation of (4).

Hydrostatic balance and gradient wind balance are assumed in the free atmosphere as well and they may be written

$$
\begin{gathered}
\alpha \frac{\partial p}{\partial z}=-g \\
\alpha \frac{\partial p}{\partial r}=\frac{V^{2}}{r}+f V
\end{gathered}
$$

where $g$ is the acceleration of gravity, $p$ the pressure and $\alpha$ the specific volume.

The cornerstone of E86 theory, which shows that knowledge of $s_{m}$ as a function of $r$ allows to determine $M$ as a function of $r$ at the TBL, may be written

$$
-r^{2} \frac{\partial s_{m}}{\partial r} \Delta T=\frac{1}{2} \frac{\partial M^{2}}{\partial r}
$$

where $\Delta T=T_{t b l}-T_{o u t}, T_{t b l}$ is the temperature at the TBL and $T_{\text {out }}$ is the temperature in the outflow region.

A key step in our work is that we propose a solution to $s_{m}$ in (8)

$$
s_{m}(r)=\Delta s_{m} e^{-\frac{r^{2}}{2 \lambda^{2}}}+s_{e n v}
$$

where $\Delta s_{m}$ is the moist entropy increment from the ambient environment to the TC centre, $s_{e n v}$ the moist entropy in the ambient environment. (9) is a Gaussian distribution, and the physical meaning of $\lambda$ here is the horizontal width of moist entropy at the TBL. This choice is motivated by the excellent Gaussian fit (more details in Section 3.2).

Assuming that the azimuthal velocity at the centre of a TC is zero, and assuming further that $\Delta T$ is a constant, by virtue of (9) one can integrate (8) to obtain,

$$
M(r)=\mu \sqrt{2 \lambda^{2}(1-\epsilon)-r^{2} \epsilon}
$$


in which $\mu=\sqrt{2 \Delta T \Delta s_{m}}$, and $e^{-\frac{r^{2}}{2 \lambda^{2}}}$ is replaced with $\epsilon$ for simplicity.

Using (5) in (7) and eliminate $M$ by virtue of (10), (7) may be written

$$
\alpha \frac{\partial p}{\partial r}=\frac{M^{2}}{r^{3}}-\frac{f^{2}}{4} r=\mu^{2}\left[\frac{2 \lambda^{2}}{r^{3}}(1-\epsilon)-\frac{1}{r} \epsilon\right]-\frac{f^{2}}{4} r
$$

Neglecting horizontal variations in $\alpha$, this equation can further be integrated with respect to radius to obtain,

$$
p(r)=\frac{\mu^{2}}{2 \alpha} \frac{1-\epsilon}{\ln \epsilon}-\frac{f^{2} r^{2}}{8 \alpha}+C
$$

in which $C$ is a constant of integration. (12) shows that the presence of TC introduces a pressure perturbation $p^{\prime}(r)$,

$$
p^{\prime}(r)=\frac{\mu^{2}}{2 \alpha} \frac{1-\epsilon}{\ln \epsilon}
$$

which, at the centre of the TC, is simply $p^{\prime}(0)=\Delta p$ where

$$
\Delta p=\frac{\mu^{2}}{2 \alpha}
$$

Note that this equation can be interpreted physically by rewriting it as $\alpha \Delta p=\Delta T \Delta s_{m}$, equating the work of expansion $(\alpha \Delta p)$ with the heat added by air sea fluxes at the sea surface times an efficiency $\left(T_{s} \Delta s_{m} \times \Delta T / T_{s}\right)$.

Using (10) and (14) in (5), one may get an analytical solution to tangential velocity at the TBL

$$
V=\sqrt{2 \alpha \Delta p} \sqrt{\frac{2 \lambda^{2}}{r^{2}}(1-\epsilon)-\epsilon}-\frac{1}{2} f r
$$

Substituting an arbitrary threshold wind velocity $V_{t h}$ in this equation and neglecting terms involving $\epsilon$ at a radius much larger than $\lambda$, (15) can be simplified as

$$
\frac{f}{2} r_{t h}^{2}+V_{t h} r_{t h}-2 \lambda \sqrt{\alpha \Delta p}=0
$$

where $r_{t h}$ is the radius defined with $V_{t h}$ at the TBL. The value of this radius is then given by solving analytically (16),

$$
r_{t h}=\frac{\sqrt{V_{t h}^{2}+4 f \lambda \sqrt{\alpha \Delta p}}-V_{t h}}{f}
$$

Equation (17) shows how the size of the TC depends on its pressure deficit $(\Delta p)$ and the width of the entropy distribution in the boundary layer $(\lambda)$, for a given wind threshold. The only scaling parameter is $\lambda$, whereas the widely used $\mathrm{H}$ model has two scaling parameters. This model for the radial wind profile is referred to in the following as the $\lambda$ model. Its prediction of TC size is illustrated in Figure 2. At fixed $\Delta p$, the size, defined by any wind threshold, increases with $\lambda$. Conversely, at fixed $\lambda$, a greater pressure deficit results in a larger TC. The $\lambda$ model makes an important prediction: for a fully developed tropical cyclone, its size largely depends on $\lambda$ and varies weakly with $\Delta p$. We test this prediction with the full-physics numerical model in the next section.

\section{Results}

\subsection{Intensity and MPI comparisons}

Figure 3 shows the time series of the minimum surface pressure $\left(p_{\text {min }}\right)$. In CTRL, the initial central pressure is about $965 \mathrm{hPa}$. $p_{\text {min }}$ decreases by almost $80 \mathrm{hPa}$ in the first three simulation days, and reaches the minimum value on the fourth simulation day. In the next four days, $p_{\min }$ reaches a relatively steady state. The pressure changes in $\mathrm{T}_{\mathrm{UT}}( \pm 2)$ (Figure 3(a)) is similar to CTRL. However, the pressure difference in $\operatorname{SST}( \pm 1)$ are significant (Figure 3(b)). The small shifts at the beginning in $\mathrm{V}_{\mathrm{m}}( \pm 5)$ (Figure 3(c)) and $R_{m}( \pm 25)$ (Figure 3(d)) are caused by the adjustment processes. The pressure in $\mathrm{V}_{\mathrm{m}}(-5)$ attains the maximum value about 24 hours later than the CTRL and $\mathrm{V}_{\mathrm{m}}(+5)$. The pressure difference between CTRL and $R_{m}( \pm 25)$ is not noticeable. Figure 4 shows the time series of the maximum wind at a height of 10 meters $\left(V_{10 \max }\right) . V_{10 \max }$ in CTRL increases by more than 40 $\mathrm{m} \mathrm{s}^{-1}$ in the first three simulation days, and attains the maximum value on the fourth simulation day. $V_{10 \max }$ tendencies in other sensitivity experiments are similar to CTRL. According to the changes of $p_{\min }$ and $V_{10 \max }$, we defined the developing stage from simulation hour 0 to 72 and the mature stage from simulation hour 73 to 192 .

The simulated intensity $V_{10 \max }$ is compared with the MPI in Table 2. According to the MPI calculation (3), $V_{m p i}$ for the environment specified with Jordan's sounding is $67 \mathrm{~ms}^{-1}$. 
In $\mathrm{V}_{\mathrm{m}}( \pm 5)$ and $\mathrm{R}_{\mathrm{m}}( \pm 25), V_{m p i}$ is the same because the environment is unchanged. The difference between $V_{m p i}$ and $V_{10 \max }$ in CTRL, $\mathrm{V}_{\mathrm{m}}( \pm 5)$ and $\mathrm{R}_{\mathrm{m}}( \pm 25)$ is smaller than $3 \mathrm{~m} \mathrm{~s}^{-1}$. $V_{m p i}$ increases in $\operatorname{SST}(+1)$ and $\mathrm{T}_{\mathrm{UT}}(-2)$. However the response in those experiments compared to CTRL is less in the model than predicted by the upper limit of MPI. $V_{m p i}$ in experiments $\operatorname{SST}(-1)$ and $\mathrm{T}_{\mathrm{UT}}(+2)$ decreases, and there is corresponding drop in $V_{10 \max }$. The response in those experiments compared to the CTRL is again much less than predicted by MPI. One consequence of this is that for the $\operatorname{SST}(-1)$ experiment $V_{m p i}$ is surprisingly less than $V_{10 \max }$.

\section{2. $\lambda$ model and the simulations}

The relevance of (9) to the numerical model entropy in CTRL at simulation hour 150 is assessed in Figure 5. The $\lambda$ model assumed a well-mixed boundary layer so the vertically averaged entropy (over the lowest six levels, approximately $600 \mathrm{~m}$ ) is calculated using (4). There is an excellent fit of the Gaussian function to the entropy distribution in the later stage of the cyclone in the control simulation (Figure 5(a)). As the Gaussian distribution of moist entropy is the foundation of the $\lambda$ model, we take a further step to verify the validity of this assumption. For comparison purpose, one of the other possible solutions to $s_{m}$ in (8) is given as an example,

$$
s_{m}(r)=\Delta s_{m} e^{-\frac{r}{\lambda}}+s_{e n v}
$$

Equation (18) is in an exponential form. By taking the natural logarithm of percentage change of $s_{m}(r),(9)$ and (18) can be transformed to parabolic and linear forms, respectively. Figure 5(b) shows that parabolic fit gives a much better result, which further supports the choice (9).

Figure 6 shows the time series of $R^{2}$ from the Gaussian fit in all the experiments. The adjustment from an initially assumed exponential distribution to a Gaussian occurs within 24 hours. The $R^{2}$ at the simulation hour 24 is close to 0.96 , and it increases slightly in the next 48 hours. There is also an excellent fit for all the experiments (mean $R^{2}=0.99$ ) from hour 48 onwards even before the cyclone is fully developed. The noise becomes stronger in the last 24 hours, but $R^{2}$ is still greater than 0.96 .
Having shown that (9) can describe the simulated moist entropy distribution we can apply this assumption to predict the speed distribution as a function of $\Delta p$ and $\lambda$ using (15). Deriving the central pressure at the TBL and using an ambient pressure of $916 \mathrm{hPa}$, figure 7 shows a good fit $\left(R^{2}=0.94\right)$ of the $\lambda$ model at the simulation hour 150 in CTRL. All essential features of the wind distribution are captured: the radial position and value of maximum wind, the dramatic increase in velocity from the centre to the eyewall and gradual decrease outside the eyewall. There is a small shift outwards near the maximum and underestimation at larger radii. With $\Delta p$ and $R M W$ from simulations, the $\mathrm{H}$ model gives a good approximation $\left(R^{2}=0.91\right)$ to the wind profile as well. However, the $\mathrm{H}$ model overestimates the wind speed outside the eyewall at this time. For the $\lambda$ model, figure 8 shows that there is also a good fit for all the experiments (mean $R^{2}=0.89$ ) from hour 72 onwards when the cyclone is fully developed. The comparison between the $\lambda$ model and $\mathrm{H}$ model in Figure 8 shows that the $\lambda$ model seems as good as the extensively used $\mathrm{H}$ model (mean $R^{2}=0.89$ ) but uses one less parameter.

\subsection{Size and $\lambda$}

We measure TC size as the radius of hurricane-force wind $(\approx$ $\left.33 \mathrm{~m} \mathrm{~s}^{-1} ; R 33\right)$, radius of damaging-force wind $\left(\approx 26 \mathrm{~m} \mathrm{~s}^{-1}\right.$; $R 26)$, radius of gale-force wind $\left(\approx 17 \mathrm{~m} \mathrm{~s}^{-1} ; R 17\right)$ and $R M W$. All the size measurements are based on azimuthally averaged tangential wind (Chan and Yip 2003; Dean et al. 2009; Xu and Wang 2010a,b). The size variations of $R 33$ and $R 17$ are similar to $R 26$ so only the results of $R 26$ and $R M W$ are shown.

Figure 9 shows that at the mature stage (simulation hour 150), clear changes in the distance of damaging-force wind occur in all sets of experiments. In addition, they are essentially axisymmetric which allows us to use the $R 26$ value. Figure 9 shows that TC size is sensitive to the upper tropospheric temperature, SST and initial vortex structure. Figure 10 depicts the time evolution of $R 26$ in all the experiments. $R 26$ in CTRL increases significantly in the developing stage, and the size becomes relatively steady after exceeding about $100 \mathrm{~km}$. Figure 10(a) shows that the $R 26$ in $\mathrm{T}_{\mathrm{UT}}(-2)$ starts to increase earlier and is always greater than that in CTRL and $\mathrm{T}_{\mathrm{UT}}(+2) \cdot R 26$ in $\mathrm{SST}( \pm 1)$ (Figure 10(b)) increases almost at the same time, but the rise of $\operatorname{SST}(+1)$ is much bigger, 
and the $R 26$ at hour 192 is about $170 \mathrm{~km}$, which is the biggest size in all the experiments. Figure 10 (c) shows that $R 26$ of $\mathrm{V}_{\mathrm{m}}(+5)$ begins to rise about 20 hours earlier than that in $\mathrm{V}_{\mathrm{m}}(-5)$, and the earlier the increase happens, the larger the final size is. However, $R 26$ begins to increase earlier in $R_{m}(-25)$ than $R_{m}(+25)$, but there is a bigger cyclone in $\mathrm{R}_{\mathrm{m}}(+25)$ at the mature stage. These time series show the same variations in TC size at the mature stage as Figure 9, which suggests that the changes in size shown in Figure 9 are persistent.

As for $R M W$ (Figure 11), the changes are noiser than the variations in $R 26$. In the first simulation day, $R M W$ drops significantly to about $30 \mathrm{~km}$, and then increases gradually to around $60 \mathrm{~km}$. The size evolution and response to the environmental conditions are similar to $R 26$ at the later stage of the development.

Figure 12 shows the time series of $\lambda$ obtained from fitting the Gaussian distribution to the simulated moist entropy. In $\mathrm{T}_{\mathrm{UT}}( \pm 2)$ and $\operatorname{SST}( \pm 1)$, the initial $\lambda$ is the same, whereas small shifts (about $\pm 4 k m)$ in $\mathrm{R}_{\mathrm{m}}( \pm 25)$ and $\mathrm{V}_{\mathrm{m}}( \pm 5)$ are generated due to changes in initial wind profiles. At the developing stage, the changes in $\lambda$ are noisy, but the amplitude of the noise decreases with time. In the mature stage, every set of experiments shows clear differences in $\lambda$ and $\lambda$ increases gradually at this stage, just like the size. Comparing Figure 12 with 10 and 11, one can see that the changes in $\lambda$ is more similar to the variations in $R M W$, and at the mature stage, there should be a positive correlation between TC size and $\lambda$.

\subsection{Linear relationship between size and $\sqrt{\lambda}$}

Based on (17), there is a predicted simple relationship for the radii and $\lambda$ in the special case $V_{t h}=0$ :

$$
r_{t h} \propto \sqrt{\lambda}
$$

We test (19) by comparing $\sqrt{\lambda}$ from entropy fitting at the mature stage in all the experiments with $R 26$, a more common TC size definition, instead of the radius of vanishing wind which is difficult to find in the simulations due to noise. Figure 13(a) shows that bigger cyclones correspond to larger $\sqrt{\lambda}\left(R^{2}=0.84\right)$. The $R^{2}$ of $R 17$ and $R 33$ with $\sqrt{\lambda}$ are 0.80 and 0.74 , respectively.
Figure 13(b) and 13(c) are shown to compare $\lambda$ to the other two fitting coefficients $\left(\Delta s_{m}\right.$ and $\left.s_{e n v}\right)$ in (9). There is no good relationship between size and the entropy increment from the ambient environment to the TC centre or the environmental entropy. Furthermore, although we did not analytically solve for $R M W$, the empirical linear relationship $\left(R^{2}=0.90\right)$ is even better than that between $R 26$ and $\sqrt{\lambda}$ (Figure 14).

\section{5. $\sqrt{\lambda}$ and angular momentum}

To further understand the physical process related to the change in size, the relationship between $\sqrt{\lambda}$ and the symmetric radial angular momentum flux (SAMF) is shown in Figure 15. SAMF is defined as the sum of symmetric relative angular momentum flux and the symmetric Coriolis torque. The reader is referred to Chan and Chan (2013) for a detailed derivation of SAMF. The SAMF here is the hourly mean flux within the boundary layer and within $R 17$ which is the most outwards size considered in this paper. A negative value means angular momentum import towards the center. Figure 15 shows a good linear relationship $\left(R^{2}=0.74\right)$ between SAMF and $\sqrt{\lambda}$. Figure 15 suggests angular momentum transport in the boundary layer is related to the change in TC size.

\section{Discussions}

We find the intensity changes are only small for the range of experiments. SST does cause certain variations in intensity after the second simulation day, but the $V_{10 \max }$ difference gradually reduces with time at the mature stage (Figure 4(b)). Figure 4(c) shows that the cyclone starting with a weak vortex results in a slightly intenser cyclone at the mature stage. However, Rotunno and Emanuel (1987) found that an initially weak vortex leads to a weaker cyclone, which is opposite to our finding. We note that the initial maximum wind speed of the weak vortex in this study is $15 \mathrm{~m} \mathrm{~s}^{-1}$, whereas the initial maximum wind speed in their work was only $2 \mathrm{~m} \mathrm{~s}^{-1}$, which may be too weak for a vortex to get fully developed.

In general, the variations in simulated intensity are consistent with the theoretical MPI results. However, compared to CTRL the response in $\operatorname{SST}( \pm 1)$ and $\mathrm{T}_{\mathrm{UT}}( \pm 2)$ is smaller than MPI predicts and this is expected as the MPI is intended as a plausible upper limit, whereas the full model includes atmospheric 
negative feedbacks such as enhanced radiation and frictional loss. $\mathrm{SST}(-1)$ produces $5 \mathrm{~ms}^{-1}$ stronger winds than the potential maximum wind speed. The cause for this is not clear however we note that the fluctuation of the maximum wind speed is about \pm 5 $\mathrm{ms}^{-1}$ in the mature state and this "superintensity" is also found by others (e.g., Persing and Montgomery 2003).

The $\lambda$ model, according to the fitting results (Figure 5, 6, 7 and 8 ), is a promising solution to describing the tangential velocity distribution. The single-parameter $\lambda$ model seems as good as the $\mathrm{H}$ model with two scaling parameters. It is also pleasing to note that there is physical basis (the thermal wind balance, (8)) in the $\lambda$ model. We have developed the model further to explore TC size. One can identify three different factors in TC size from (17). Firstly, the pressure drop from the ambient environment to the eye, secondly the Coriolis parameter, and thirdly the distribution of moist entropy at the TBL given by $\lambda$. According to (17) the cyclone should shrink with increasing latitude. To support this argument, an additional set of simulations were performed. We ran the CTRL experiment on the f-plane at $20^{\circ} \mathrm{N}, 30^{\circ} \mathrm{N}$ and $40^{\circ} \mathrm{N}$, and the results show that the average $R 26$ within the mature stage are $109 \mathrm{~km}, 98 \mathrm{~km}$ and 94km, respectively. This result shows the qualitative agreement with (17). However, we note that the change in $R 26$ caused by varying $f$ is only half or less than (17) suggests for the radius of vanishing wind. The changes in $R 17$ and $R 33$ with latitude are similar to those of R26. In a model study Chavas and Emanuel (2014) also found that the radius of vanishing wind increases nearly linearly with $1 / f$. Dean et al. (2009) shows that according to E86 theory the upper limitation of the overall storm size is proportional to $1 / f$. However, these are not consistent with other observational studies (Weatherford and Gray 1988; Kimball and Mulekar 2004), which suggest that TCs at higher latitude have greater size. Interestingly, Smith et al. (2011) and Chan and Chan (2014a) show that an optimum region may exist for a TC to attain a maximum size. To investigate the reason for those disagreements, more simulation experiments would need to be performed.

One should be aware of that the starting assumption of this model is a solution to the moist entropy distribution at the TBL. The assumed initial moist entropy is in an exponential distribution, and in the developing stage, entropy distribution turns into Gaussian form in less than 24 hours. Since this
Gaussian solution to the moist entropy is similar to the solution to the one-dimensional diffusion equation, we speculate that the process, in which the distribution of moist entropy turns from an exponential to a Gaussian form, is dominated by horizontally turbulent diffusion in the boundary layer down the gradient of high entropy to low entropy. This might be represented by a diffusivity coefficient through a flux gradient relationship. However, we found that the hourly eddy radial entropy flux has no relationship with $\lambda$ so this explanation of the Gaussian shape appears unlikely.

We have identified that the TC size is sensitive to environmental temperature and initial vortex structure (Figures 9, 10 and 11). Cold upper troposphere, warm SST, a large and intense initial vortex are all favourable to TC size growth. By altering uppertropospheric (150-300 $\mathrm{hPa}$ ) temperature, the outflow temperature is changed. E86 briefly indicates that outflow temperature may be negatively related to the radius of outermost closed isobar. Although we use a different size definition, the results agree. This relationship between size and upper-tropospheric temperature suggests that there could be trends in the size of TCs not just the intensity (Emanuel et al. 2013). Increased SST creates more significant air-sea temperature contrast and it results in stronger latent energy transfer from the ocean to air as found by Radu et al. (2014). The cyclone size is sensitive to the initial vortex structure. This finding is in agreement with the model simulations by $\mathrm{Xu}$ and Wang (2010a) and Chan and Chan (2014a,b). However, it should be noted that by changing the initial $R M W$ or $V_{m}$ the $B$ value also changes in the $\mathrm{H}$ model (Figure 1(b)), which means the whole wind profile changes. That causes a compounded effect of inner and outer-core winds on TC size, namely, one cannot conclude inner-core wind structure is more important to size than the outer one based on just these experiments $\left(\mathrm{R}_{\mathrm{m}}( \pm 25)\right.$ and $\left.\mathrm{V}_{\mathrm{m}}( \pm 5)\right)$. Nevertheless the $\lambda$ model is a good description of the mature stages in all these experiments, which is the main finding of our work.

Although the SST is held constant throughout the simulation so that the TC has a constant energy reservoir, the intensity $\left(V_{10 \max }\right)$ does decrease slightly (Figure 4$)$. In contrast to this the cyclone size continues to increase in all the experiments (Figure 10 and 11). A possible explanation is that, the increase in TC size results in a reduction in intensity through angular momentum 
conservation. According to MPI theory, there is an upper limit of TC intensity for this environment. However, it seems plausible that such limit does not exist for TC size as long as the stable energy supply exists.

For all the experiments the changes in intensity are smaller and sometimes hardly noticeable. However the changes in TC size are much larger and clearer. This suggests only a weak relationship between intensity and size. The $\lambda$ model provides a potentially theoretical explanation of this from equation (17) when $V_{t h}=0$,

$$
r_{t h} \propto \sqrt[4]{\Delta p}
$$

One can see that the radius of vanishing wind is only proportional to the 4 th root of the pressure deficit, whereas the dependence on the width of the entropy distribution is proportional to $\sqrt{\lambda}$. A similar prediction of $R 26$ is shown in Figure 2 and also supported by previous climatological studies showing that size is only weakly correlated with intensity (Frank and Gray 1980; Merrill 1984; Weatherford and Gray 1988; Chan and Chan 2012).

The assumed entropy depends on three variables in (9): the environmental entropy, the difference in entropy between the environment and the core, and the width of the entropy distribution, $\lambda$. However, only $\lambda$ shows clear impact on the size not the entropy in the ambient environment or the contrast between the environment and the central areas (Figure 13). The entropy distribution for the different experiments mainly changes in the central area with a radius of less than $100 \mathrm{~km}$ (Figure 5). This may be the reason that $\lambda$ has a closer relationship to $R M W$ than $R 26$.

Another explanation of the good relationship between $\lambda$ and $R M W$ can be explored by expanding the theoretical analysis. Taking the derivative of tangential wind in (15) with respect of $r$ and making it equal to zero, one obtains

$$
\begin{aligned}
& {\left[\epsilon_{m}\left(\frac{R M W}{\lambda^{2}}+\frac{2}{r}+4 \frac{\lambda^{2}}{R M W^{3}}\right)-4 \frac{\lambda^{2}}{R M W^{3}}\right]^{2} } \\
= & \frac{f^{2}}{\mu^{2}}\left[\frac{2 \lambda^{2}}{R M W^{2}}-\left(\frac{2 \lambda^{2}}{R M W^{2}}+1\right) \epsilon_{m}\right]
\end{aligned}
$$

where $\epsilon_{m}=e^{-\frac{R M W^{2}}{2 \lambda^{2}}}$.
According to the scale analysis with the typical values of $R M W\left(\sim 10^{4} \mathrm{~m}\right), f\left(\sim 10^{-4} \mathrm{~s}^{-1}\right), \mu\left(\sim 10^{2} \mathrm{~J}^{\frac{1}{2}} \mathrm{~kg}^{-\frac{1}{2}}\right)$ and $\lambda$ $\left(\sim 10^{4} \mathrm{~m}\right)$, r.h.s part of the above equation can be neglected and (21) can be written

$$
\epsilon_{m}\left(\frac{R M W}{\lambda^{2}}+\frac{2}{R M W}+4 \frac{\lambda^{2}}{R M W^{3}}\right)-4 \frac{\lambda^{2}}{R M W^{3}}=0
$$

Equation (22) has no analytic solution. However it does indicate that $R M W$ is a function of $\lambda$ alone. In contrast $R 26$ depends on $\lambda$ and albeit weakly on $\Delta p$. This may further explain the better relationship between $\lambda$ and $R M W$ than for $R 26$.

Figures 13(a) and 14 show that TC size is well correlated to $\sqrt{\lambda}$, which suggests we can understand size in terms of $\sqrt{\lambda} \cdot \sqrt{\lambda}$ is a property of moist entropy distribution at TBL and according to our derivation starting from E86 the moist entropy is a function of angular momentum per unit mass. This means TC size should be highly related to the radial angular momentum distribution and transport near the boundary layer. This argument agrees with Chan and Chan (2013) who showed that based on observations and reanalysis data the SAMF in lower troposphere is important to the change in TC size. As for our simulations, the good correlation between $\sqrt{\lambda}$ and SAMF in the boundary layer shown in Figure 15 further supports the connection between the change in TC size and the angular momentum transport in the boundary layer.

\section{Conclusions}

A new analytic tropical cyclone model, $\lambda$ model, has been derived. The $\lambda$ model correctly depicts the tangential velocity profile at the TBL. Based on the $\lambda$ model, the TC size is a function of the distribution of moist entropy at the TBL given by $\lambda$, the pressure drop from the ambient environment to the eye, and the Coriolis parameter. In the simulations, we found that SST, upper troposphere temperature and initial vortex structure can all affect the subsequent TC size. These size changes caused by different factors all have good relationships with the width of a Gaussian moist entropy distribution as shown by the $\lambda$ model.

With regards to TC size and intensity, we find that, unlike the intensity prediction based on the MPI theory, it seems that there is no upper limit for TC size provided there is sufficient energy support from the ocean. The increase in TC size at the mature 
stage also causes a slight drop in intensity. In addition, a weak relationship between TC size and intensity is confirmed in the simulations, and this relationship can be understood with the $\lambda$ model.

One may be able to predict TC size by understanding what sets the width, $\lambda$. As E86 shows that the moist entropy is a function of angular momentum per unit mass, we show that size may be highly related to angular momentum import within the boundary layer, which is consistent with observations reported by Chan and Chan (2013). On a f-plane angular momentum flux is controlled by the local angular momentum and radial wind, and the latter one may be the key factor in TC size change. The relatively simple single scaling parameter, $\lambda$ model, presented appears to capture these essential features in a physical manner.

\section{Acknowledgement}

We thank the CSC Imperial Scholarship Programme and BP Environmental Technology Programme for their support as well as the referees for their helpful comments.

\section{References}

Brand S. 1972. Very large and very small typhoons of the western North Pacific Ocean. J. Meteor. Soc. Japan 50(4): 332-341.

Chan JCL, Williams RT. 1987. Analytical and Numerical Studies of the BetaEffect in Tropical Cyclone Motion. Part 1: Zero Mean Flow. Journal of the Atmospheric Sciences 44: 1257-1265.

Chan JCL, Yip CKM. 2003. Interannual variations of tropical cyclone size over the western North Pacific. Geophysical Research Letters 30(24), doi: 10.1029/2003GL018522.

Chan KTF, Chan JCL. 2012. Size and Strength of Tropical Cyclones as Inferred from QuikSCAT Data. Monthly Weather Review 140(3): 811-824.

Chan KTF, Chan JCL. 2013. Angular Momentum Transports and Synoptic Flow Patterns Associated with Tropical Cyclone Size Change. Monthly Weather Review 141(11): 3985-4007.

Chan KTF, Chan JCL. 2014a. Impacts of initial vortex size and planetary vorticity on tropical cyclone size. Quarterly Journal of the Royal Meteorological Society 140(684): 2235-2248.

Chan KTF, Chan JCL. 2014b. Impacts of vortex intensity and outer winds on tropical cyclone size. Quarterly Journal of the Royal Meteorological Society doi:10.1002/qj.2374.

Chavas DR, Emanuel K. 2014. Equilibrium Tropical Cyclone Size in an Idealized State of Axisymmetric RadiativeConvective Equilibrium*. Journal of the Atmospheric Sciences 71(5): 1663-1680.
Cocks SB, Gray WM. 2002. Variability of the outer wind profiles of western North Pacific typhoons: Classifications and techniques for analysis and forecasting. Monthly weather review 130(8): 1989-2005.

Dean L, Emanuel KA, Chavas DR. 2009. On the size distribution of Atlantic tropical cyclones. Geophysical Research Letters 36(14), doi:10. 1029/2009GL039051.

Dudhia J. 1989. Numerical Study of Convection Observed during the Winter Monsson Experiment Using a Mesoscale Two-Dimensional Model. Journal of the Atmospheric Sciences 46: 3077-3107.

Emanuel K, Solomon S, Folini D, Davis S, Cagnazzo C. 2013. Influence of Tropical Tropopause Layer Cooling on Atlantic Hurricane Activity. Journal of Climate 26(7): 2288-2301.

Emanuel KA. 1995. Sensitivity of tropical cyclones to surface exchange coefficients and a revised steady-state model incorporating eye dynamics. Journal of the Atmospheric Sciences 52(22): 3969-3976.

Frank WM, Gray WM. 1980. Radius and Frequency of $30 \mathrm{kt}$ winds around tropical cyclones. Journal of Applied Meteorology 19: 219-223.

Hill KA, Lackmann GM. 2009. Influence of Environmental Humidity on Tropical Cyclone Size. Monthly Weather Review 137(10): 3294-3315.

Hill KA, Lackmann GM. 2011. The Impact of Future Climate Change on TC Intensity and Structure: A Downscaling Approach. Journal of Climate 24(17): 4644-4661.

Holland G. 1980. An analytic model of the wind and pressure profiles in hurricanes. Monthly weather review 108: 1212-1218.

Hong SY, Lim JOJ. 2006. The WRF Single-Moment 6-Class Microphysics Scheme (WSM6). Journal of the Korean Meteorological Society 42: 129151 .

Jordan C. 1958. Mean soundings for the West Indies area. Journal of Meteorology 15: 91-97.

Kimball SK, Mulekar MS. 2004. A 15-Year Climatology of North Atlantic Tropical Cyclones. Part I: Size Parameters. Journal of Climate 17(18): $3555-3575$

Kwok JHY, Chan JCL. 2005. The influence of uniform flow on tropical cyclone intensity change. Journal of the Atmospheric Sciences 62(9): 3193 3212 .

Lawrence MB, Avila LA, Beven JL, Franklin JL, Guiney JL, Pasch RJ. 2001. Atlantic hurricane season of 1999. Monthly Weather Review 129(12): 30573084 .

Liu KS, Chan JCL. 1999. Size of Tropical Cyclones as Inferred from ERS-1 and ERS-2 Data. Monthly Weather Review 127: 2992-3001.

Merrill RT. 1984. A Comparison of Large and Small Tropical Cyclones. Monthly Weather Review 112: 1408-1418.

Mlawer EJ, Taubman SJ, Brown PD, Iacono MJ, Clough SA. 1997. Radiative transfer for inhomogeneous atmospheres: RRTM, a validated correlated$\mathrm{k}$ model for the longwave. Journal of Geophysical Research 102(D14): $16663-16682$. 
Pauluis O, Czaja A, Korty R. 2010. The Global Atmospheric Circulation in Moist Isentropic Coordinates. Journal of Climate 23(11): 3077-3093.

Persing J, Montgomery MT. 2003. Hurricane Superintensity. Journal of the Atmospheric Sciences 60(19): 2349-2371.

Phibbs S, Toumi R. 2014. Modeled dependence of wind and waves on ocean temperature in tropical cyclones. Geophysical Research Letters 41(20): 7383-7390.

Radu R, Toumi R, Phau J. 2014. Influence of atmospheric and sea surface temperature on the size of hurricane Catarina. Quarterly Journal of the Royal Meteorological Society 140(682): 1778-1784.

Rotunno R, Emanuel K. 1987. An Air-Sea Interaction Theory for Tropical Cyclones. Part2: Evolutionary Study Using a Nonhydrostatic Axisymmetric Numerical Model. Journal of the Atmospheric Sciences 44: 542-561.

Simpson RH, Saffir H. 1974. The hurricane disaster potential scale. Weatherwise 27(8): 169.

Smith RK, Schmidt CW, Montgomery MT. 2011. An investigation of rotational influences on tropical-cyclone size and intensity. Quarterly Journal of the Royal Meteorological Society 137(660): 1841-1855.

Tiedtke M. 1989. A Comperhensive Mass Flux Scheme for Cumulus Parameterization in Large-scale Models. Monthly Weather Review 117: 1779-1800.

Wang Y. 2009. How Do Outer Spiral Rainbands Affect Tropical Cyclone Structure and Intensity? Journal of the Atmospheric Sciences 66(5): 12501273.

Weatherford CL, Gray WM. 1988. Typhoon Structure as Revealed by Aircraft Reconnaissance. Part II. Structural Variability. Monthly Weather Review 116: 1044-1056.

Xu J, Wang Y. 2010a. Sensitivity of the Simulated Tropical Cyclone InnerCore Size to the Initial Vortex Size*. Monthly Weather Review 138(11): 4135-4157.

Xu J, Wang Y. 2010b. Sensitivity of Tropical Cyclone Inner-Core Size and Intensity to the Radial Distribution of Surface Entropy Flux. Journal of the Atmospheric Sciences 67(6): 1831-1852.

Zhang C, Wang Y, Hamilton K. 2011. Improved Representation of Boundary Layer Clouds over the Southeast Pacific in ARW-WRF Using a Modified Tiedtke Cumulus Parameterization Scheme. Monthly Weather Review 139(11): 3489-3513. 
Table 1. List of simulation names for sensitivity experiments. The increments are compared to CTRL.

\begin{tabular}{ccccc}
\hline Simulation name & $\begin{array}{c}\Delta S S T \\
\left({ }^{\circ} \mathrm{C}\right)\end{array}$ & $\begin{array}{c}\Delta T_{U T} \\
\left({ }^{\circ} \mathrm{C}\right)\end{array}$ & $\begin{array}{c}\Delta V_{m} \\
\left(\mathrm{~m} \mathrm{~s}^{-1}\right)\end{array}$ & $\begin{array}{c}\Delta R_{m} \\
(\mathrm{~km})\end{array}$ \\
\hline $\mathrm{SST}(+1)$ & +1 & 0 & 0 & 0 \\
$\mathrm{SST}(-1)$ & -1 & 0 & 0 & 0 \\
$\mathrm{~T}_{\mathrm{UT}}(+2)$ & 0 & +2 & 0 & 0 \\
$\mathrm{~T}_{\mathrm{UT}}(-2)$ & 0 & -2 & 0 & 0 \\
$\mathrm{~V}_{\mathrm{m}}(+5)$ & 0 & 0 & +5 & 0 \\
$\mathrm{~V}_{\mathrm{m}}(-5)$ & 0 & 0 & -5 & 0 \\
$\mathrm{R}_{\mathrm{m}}(+25)$ & 0 & 0 & 0 & +25 \\
$\mathrm{R}_{\mathrm{m}}(-25)$ & 0 & 0 & 0 & -25 \\
\hline
\end{tabular}

13

14

15

16

17

18

19

20

21

22

23

24

25

26

27

28

29

30

31

32

33

34

35

36

37

38

39

40

41

42

43

44

45

46

47

48

49

50

51

52

53

54

55

56

57

58

59

60 
Table 2. Comparison of maximum wind speed (unit: $\mathrm{m} \mathrm{s}^{-1}$ ) between MPI theory $\left(V_{m p i}\right)$ and simulation results $\left(V_{10 \max }\right)$.

\begin{tabular}{cccc}
\hline Simulation name & $V_{\text {mpi }}$ & $V_{10 \max }$ & $V_{\text {mpi }}-V_{10 \max }$ \\
\hline $\mathrm{CTRL}$ & 67 & 65 & +2 \\
$\mathrm{~V}_{\mathrm{m}}(+5)$ & 67 & 65 & +2 \\
$\mathrm{~V}_{\mathrm{m}}(-5)$ & 67 & 65 & +2 \\
$\mathrm{R}_{\mathrm{m}}(+25)$ & 67 & 64 & +3 \\
$\mathrm{R}_{\mathrm{m}}(-25)$ & 67 & 66 & +1 \\
$\mathrm{SST}(+1)$ & 76 & 69 & +7 \\
$\mathrm{SST}(-1)$ & 57 & 62 & -5 \\
$\mathrm{~T}_{\mathrm{UT}}(+2)$ & 66 & 65 & +1 \\
$\mathrm{~T}_{\mathrm{UT}}(-2)$ & 68 & 66 & +2 \\
\hline
\end{tabular}

13

14

15

16

17

18

19

20

21

22

23

24

25

26

27

28

29

30

31

32

33

34

35

36

37

38

39

40

41

42

43

44

45

46

47

48

49

50

51

52

53

54

55

56

57

58

59

60 
Figure 1. Initial set-up of (a) air temperature soundings, (b) the speed and radial

position of maximum wind in CTRL, $V_{m}( \pm 5)$ and $R_{m}( \pm 25)$ experiments.
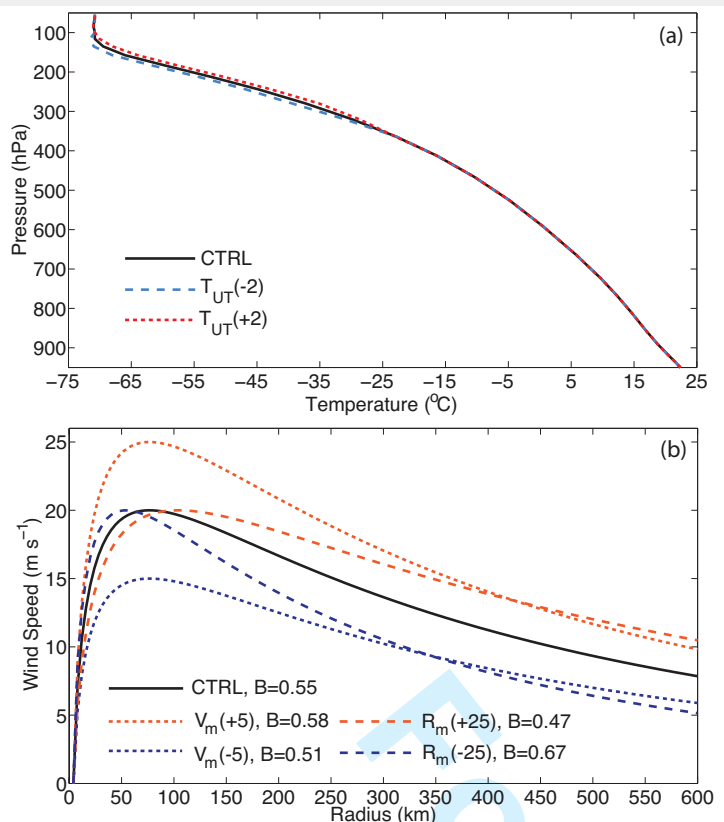
1

2

3

4

5

6

7

8

9

10

11

12

13

14

15

16

17

18

19

20

21

22

23

24

25

26

27

28

29

30

31

32

33

34

35

36

37

38

39

40

41

42

43

44

45

46

47

48

49

50

51

52

53

54

55

56

57

58

59

60

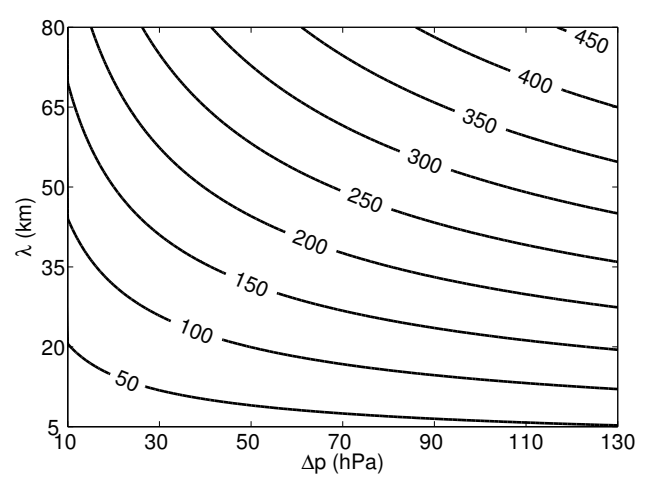

Figure 2. Radius of damaging-force wind $(\mathrm{km})$ as a function of horizontal width

$(\lambda, \mathrm{km})$ of moist entropy at the TBL and pressure deficit $(\Delta p, \mathrm{hPa})$ computed from (17) for $V_{t h}=26 \mathrm{~m} \mathrm{~s}^{-1}, f=5 \times 10^{-5} \mathrm{~s}^{-1}$ and $\alpha=0.91 \mathrm{~m}^{3} \mathrm{~kg}^{-1}$. 

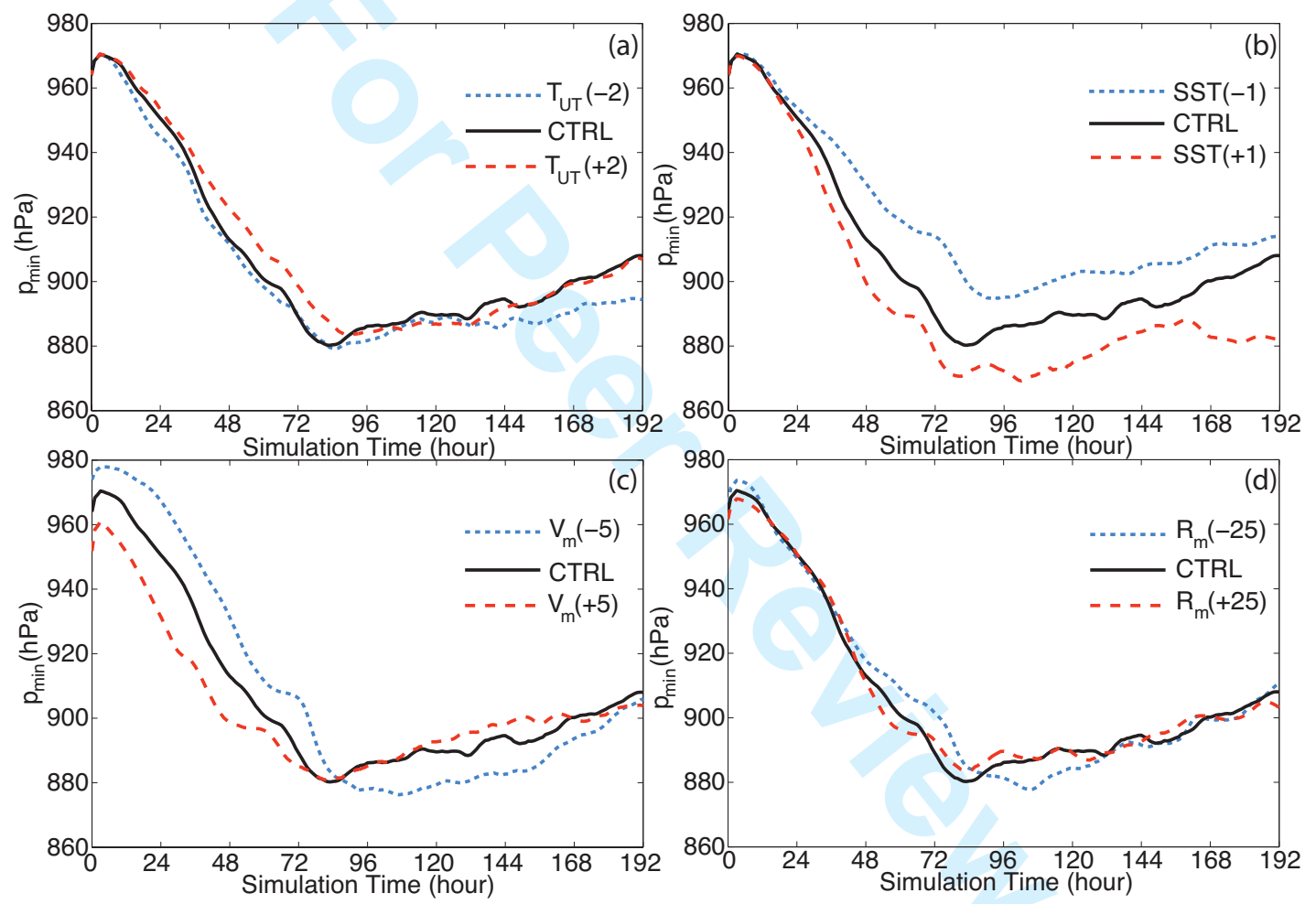

Figure 3. Time series of minimum surface pressure for (a) $\mathrm{T}_{\mathrm{UT}}( \pm 2)$, (b) $\operatorname{SST}( \pm 1),\left(\right.$ c) $\mathrm{V}_{\mathrm{m}}( \pm 5)$ and (d) $\mathrm{R}_{\mathrm{m}}( \pm 25)$ experiments, with application of a moving average smoother. The span for the moving average is 5 . 

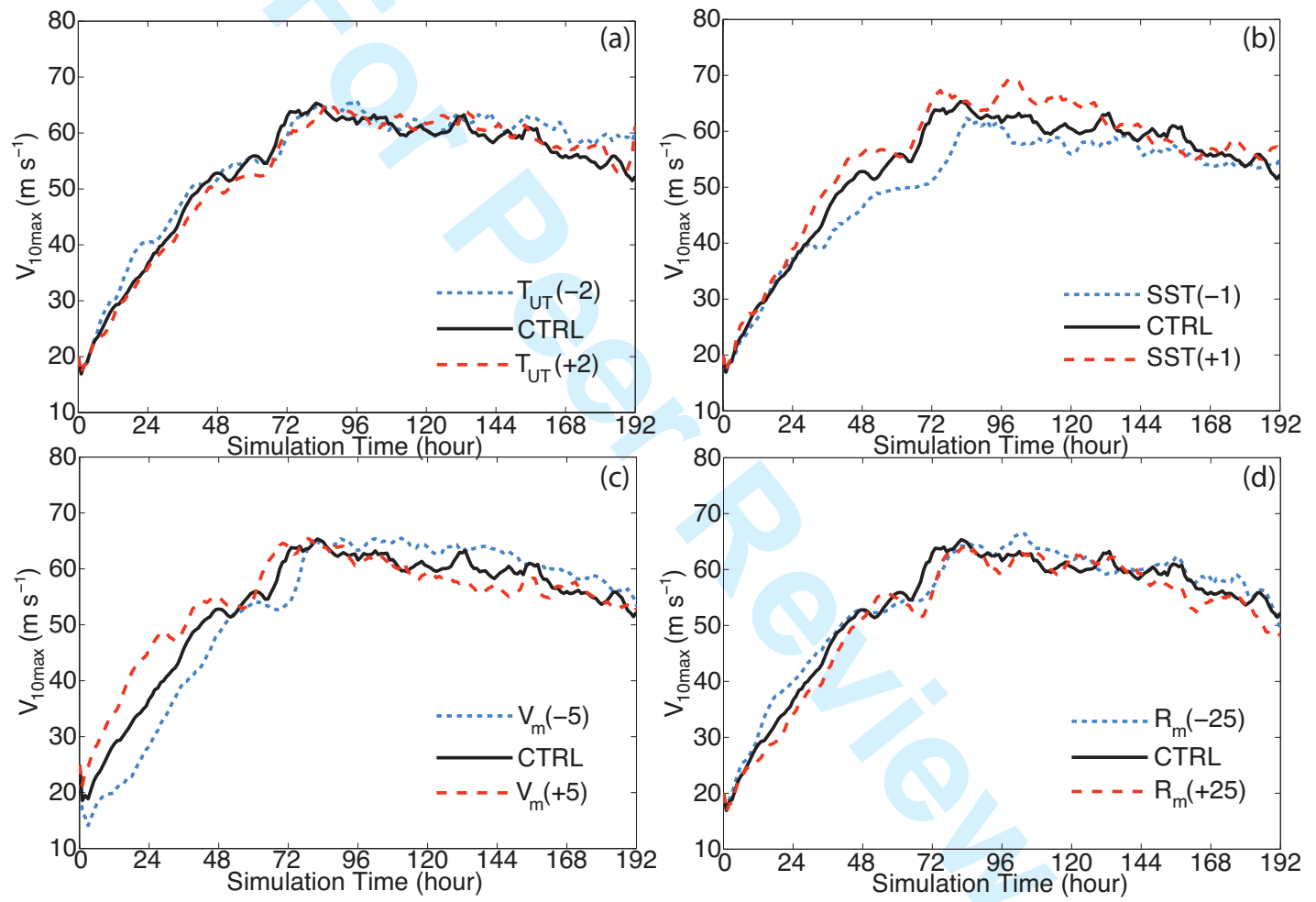

Figure 4. As in Figure 3, but for maximum wind speed. 

and linear fits of the natural logarithm of percentage change of moist entropy in CTRL at simulation hour 150. In the Gaussian fit, $\Delta s_{m}=59.6 \mathrm{~J} \mathrm{~K}^{-1} \mathrm{~kg}^{-1}, \lambda=$ $25.8 \mathrm{~km}$, and $s_{\text {env }}=290.7 \mathrm{~J} \mathrm{~K}^{-1} \mathrm{~kg}^{-1}$. In the exponential fit, $\Delta s_{m}=80.0$ $\mathrm{J} \mathrm{K}^{-1} \mathrm{~kg}^{-1}, \lambda=32.8 \mathrm{~km}$, and $s_{\text {env }}=286.1 \mathrm{~J} \mathrm{~K}^{-1} \mathrm{~kg}^{-1}$. In the parabolic and linear fit, $\lambda=26.8 \mathrm{~km}$ and $20.1 \mathrm{~km}$, respectively, and $\ln \left\{\left(s_{m}-s_{\text {env }}\right) / \Delta s\right\}$ is calculated from simulation results. 
1

2

3

4

5

6

7

8

9

10

11

12

13

14

15

16

17

18

19

20

21

22

23

24

25

26

27

28

29

30

31

32

33

34

35

36

37

38

39

40

41

42

43

44

45

46

47

48

49

50

51

52

53

54

55

56

57

58

59

60

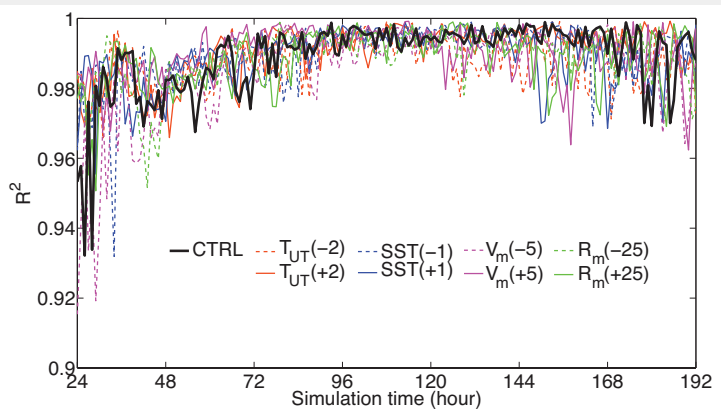

Figure 6. $R^{2}$ from entropy fitting from simulation hour 24 to 192 . 


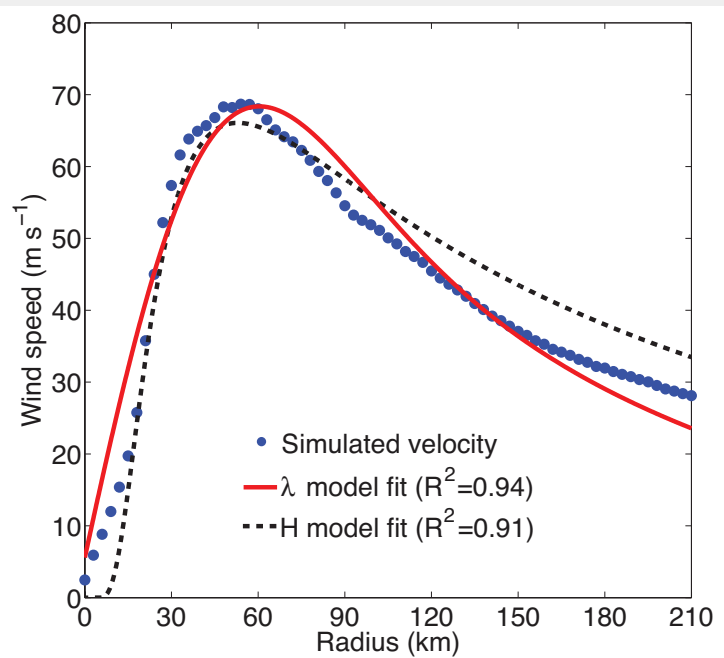

Figure 7. Fit of tangential velocity at the TBL in CTRL at simulation hour 150. In the $\lambda$ model $\lambda=34.0 \mathrm{~km}$ and in the $\mathrm{H}$ model $B=1.5$. The air density is fixed at $1.1 \mathrm{~kg} \mathrm{~m}^{-3}$ and ambient pressure at the TBL is $916 \mathrm{hPa}$. 
1

2

3

4

5

6

7

8

9

10

11

12

13

14

15

16

17

18

19

20

21

22

23

24

25

26

27

28

29

30

31

32

33

34

35

36

37

38

39

40

41

42

43

44

45

46

47

48

49

50

51

52

53

54

55

56

57

58

59

60

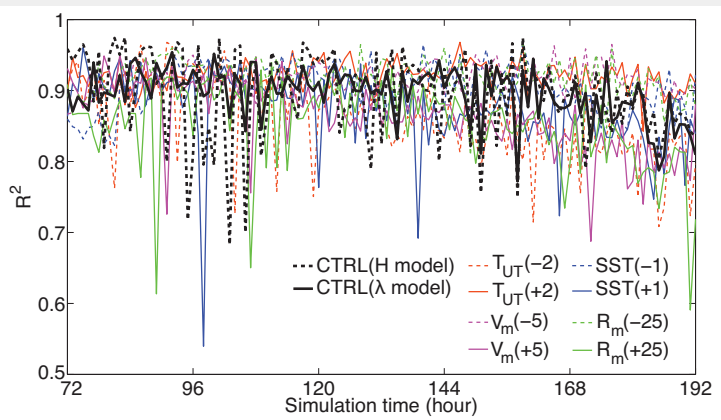

Figure 8. $R^{2}$ from tangential velocity fit at the TBL from simulation hour 72 to 192. Black dash line represents $R^{2}$ by using $\mathrm{H}$ model as a fitting function. All other lines show $R^{2}$ with (17) in $\lambda$ model as a fitting function. The central pressure and radius of maximum wind are calculated at each time steps. 

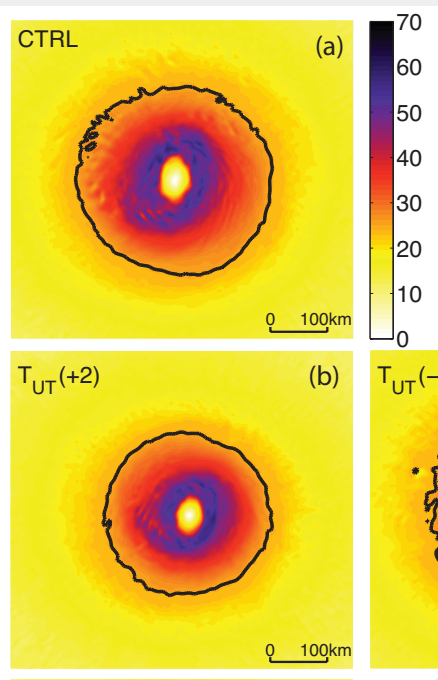

(b) $\mathrm{T}_{\mathrm{UT}}(-2)$

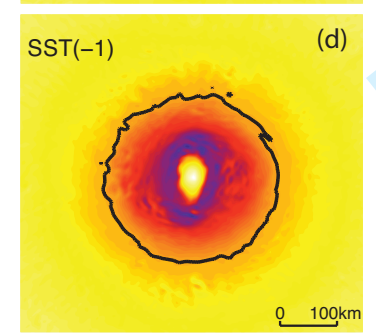

(d)
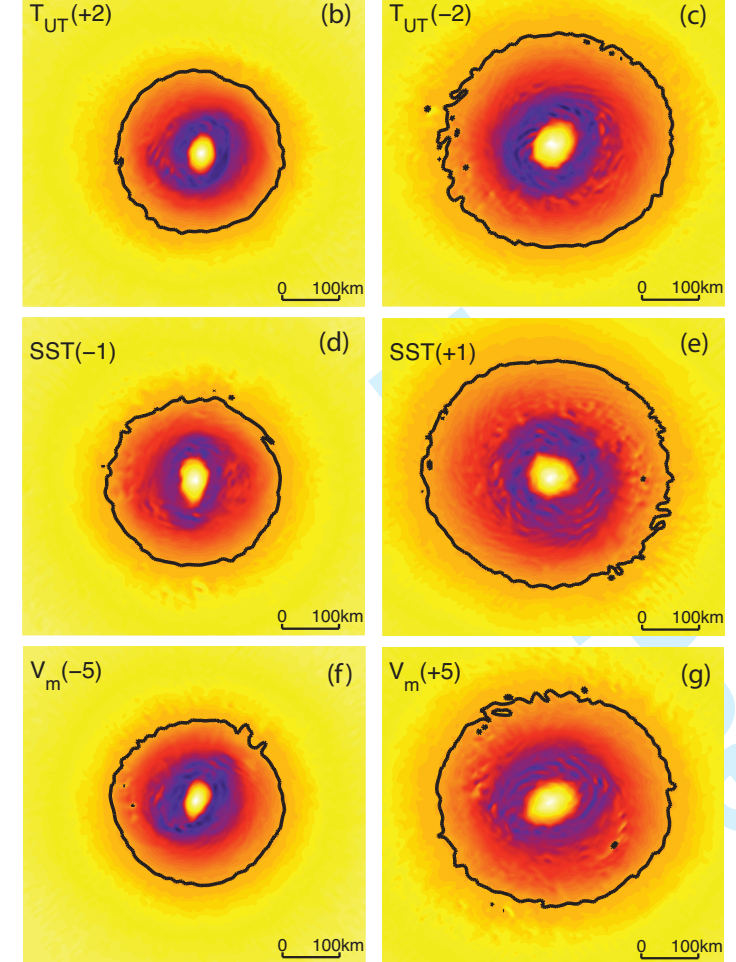

(f)
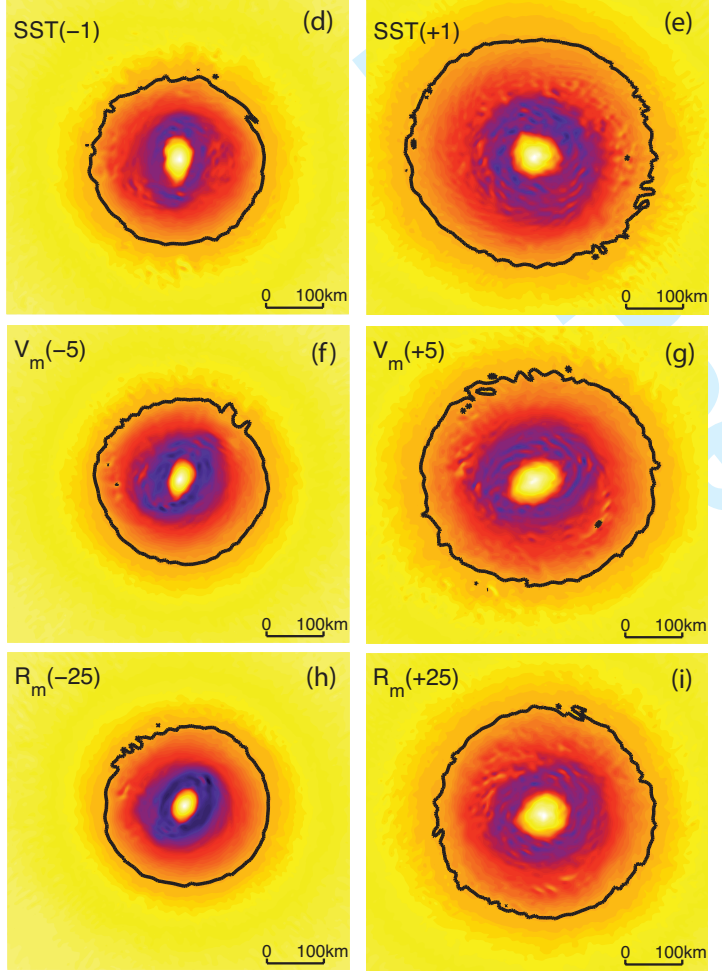

Figure 9. Model-simulated wind speed $\left(\mathrm{m} \mathrm{s}^{-1}\right)$ at a height of 10 meters at hour 144 for (a) CTRL, (b) $\mathrm{T}_{\mathrm{UT}}(+2)$, (c) $\mathrm{T}_{\mathrm{UT}}(-2)$, (d) $\operatorname{SST}(-1)$, (e)SST $(+1)$, (f) $\mathrm{V}_{\mathrm{m}}(-5)$, (g) $\mathrm{V}_{\mathrm{m}}(+5)$, (h) $\mathrm{R}_{\mathrm{m}}(-25)$ and (i) $\mathrm{R}_{\mathrm{m}}(+25)$. The black contours show the TC size defined with $R 26$. All the figures are with the same horizontal scale. 

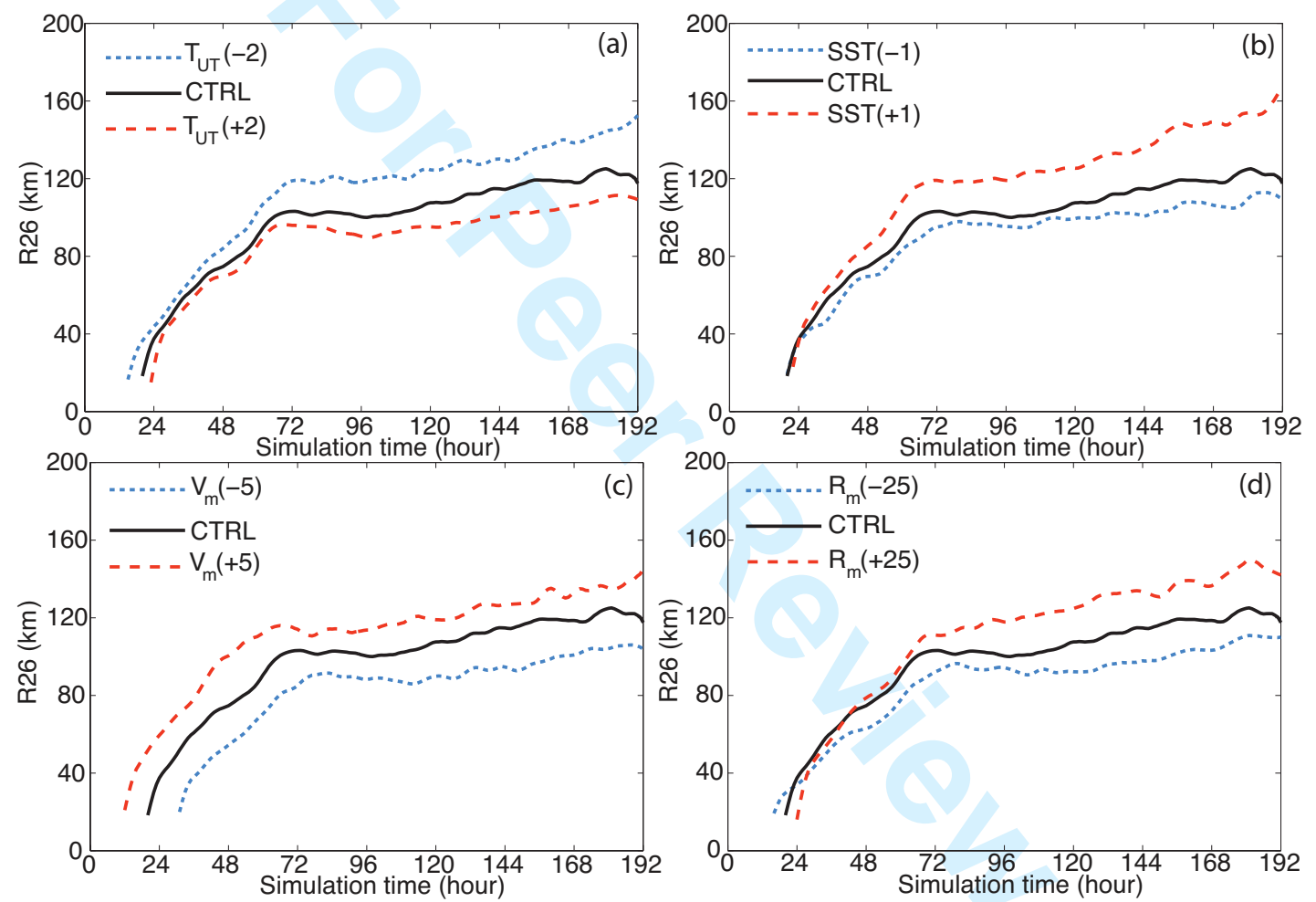

Figure 10. As in Figure 3, but for radius of damaging-force wind. 

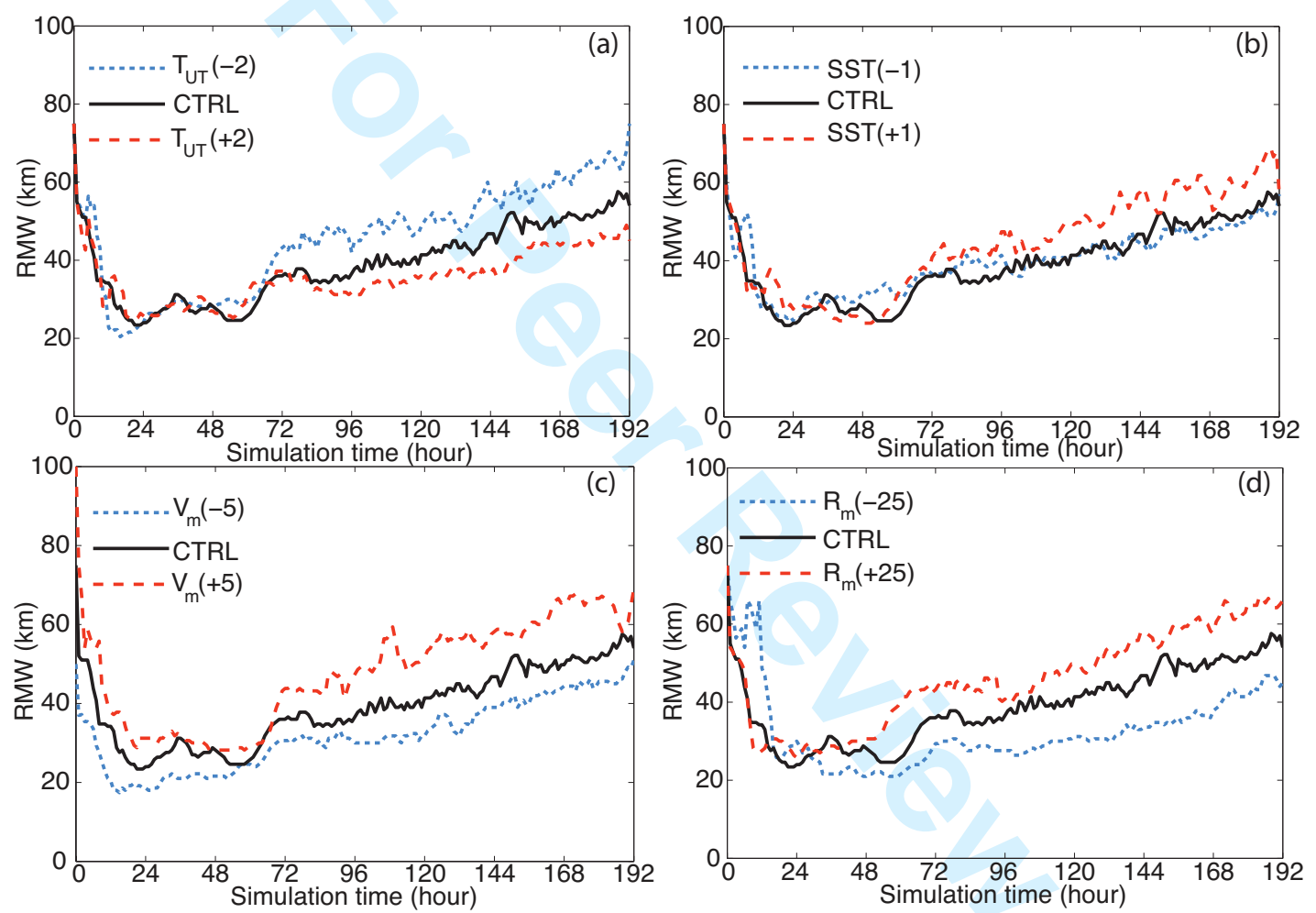

Figure 11. As in Figure 3, but for radius of maximum wind. 

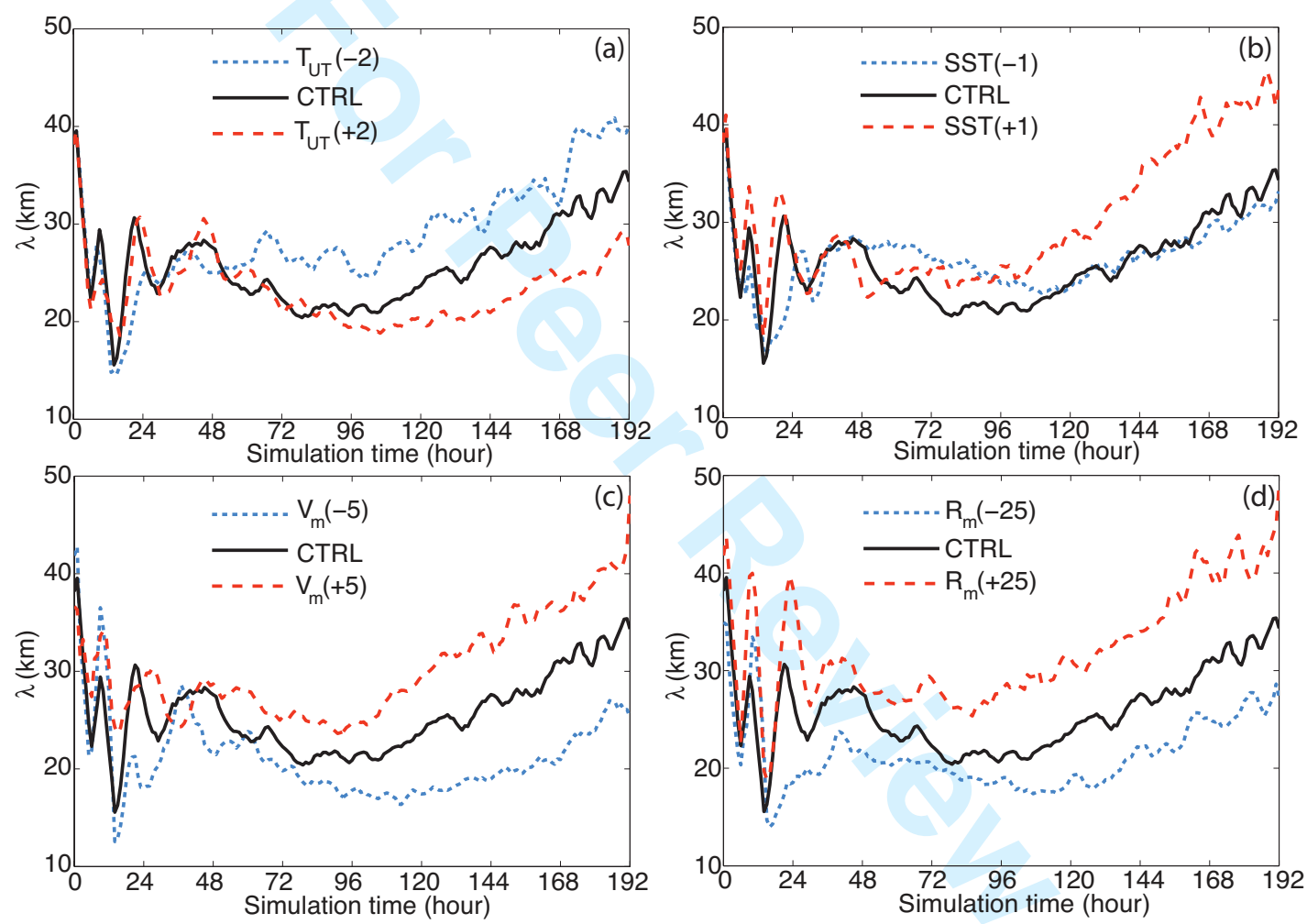

Figure 12. As in Figure 3, but for $\lambda$ from moist entropy fitting with (9) 
1

2

3

4

5

6

7

8

9

10

11

12

13

14

15

16

17

18

19

20

21

22

23

24

25

26

27

28

29

30

31

32

33

34

35

36

37

38

39

40

41

42

43

44

45

46

47

48

49

50

51

52

53

54

55

56

57

58

59

60

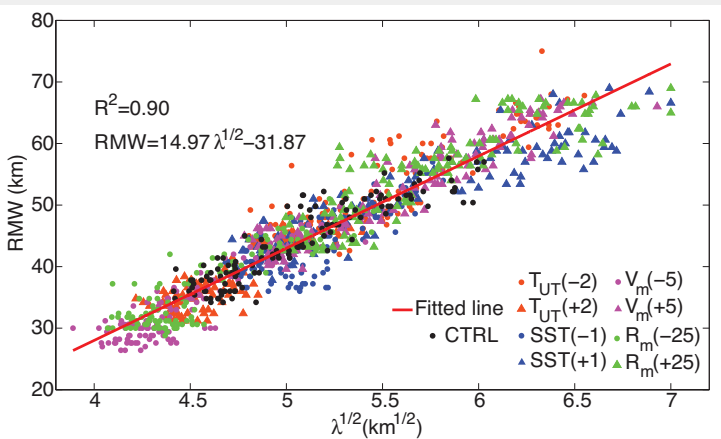

Figure 14. As in Figure 13, but for radius of maximum wind. 


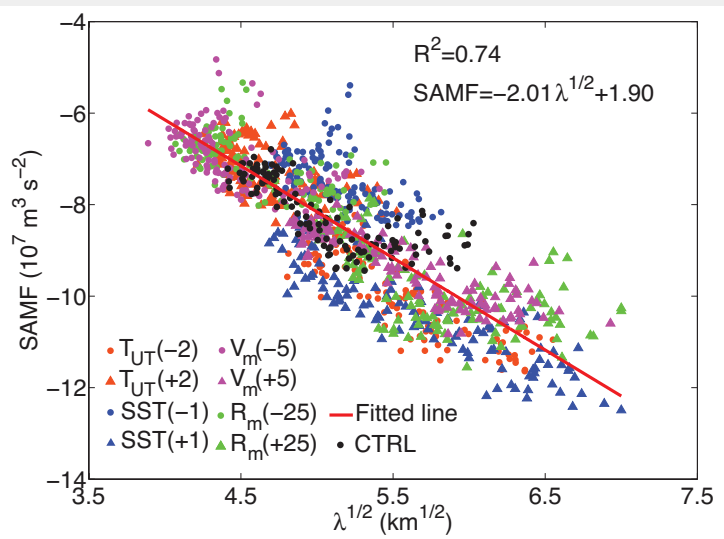

Figure 15. As in Figure 13, but for the symmetric radial angular momentum flux in the boundary layer. 